Der von der Kl. geltend gemachte grundsätzliche Klärungsbedarf ergibt sich auch nicht mit Blick auf die von ihr in Bezug genommene Entscheidung des BVerfG v. 28.8.2007 - 1 BvR 1098/07 -. Das BVerfG hat dort zwar Zweifel hinsichtlich der Auslegung des Merkmals der Unwürdigkeit durch die verwaltungsgerichtliche Rechtsprechung geäußert, weil möglicherweise verfassungsrechtlich die Prüfung geboten sei, ob von dem Betroffenen prognostisch eine Gefahr für die Allgemeinheit ausgehe (BVerfG, Kammerbeschl. v. 28. 8.2007 - 1 BvR 1098/07 -, BVerfGK 12, 72, 78). Im Kammerbeschl. v. 8.9. 2017 (1 BvR 1657/17 -, GesR 2017, 739, 740) hat es allerdings ausgeführt, dass der Bedeutung und Tragweite von Art. 12 Abs. 1 GG bei der Auslegung des Begriffs der Unwürdigkeit hinreichend Rechnung getragen wird, wenn nicht nur auf das jeweilige Fehlverhalten, sondern auch auf mögliche veränderte Umstände abgestellt wird, die eine abweichende Beurteilung der Berufsunwürdigkeit rechtfertigen könnten. Der VGH hat diese Prüfung - wie gezeigt - vorgenommen. Die Kl zeigt nicht auf, welcher weitergehende fallübergreifende Klärungsbedarf danach bestehen könnte.

2. Die Revision ist auch nicht wegen Divergenz nach $\$ 132$ Abs. 2 Nr. 2 VwGO zuzulassen. Die behauptete Abweichung des Berufungsurteils von Entscheidungen des BVerfG liegt nicht vor. [...]

https://doi.org/10.1007/s00350-020-5626-8

\section{Anmerkung zu BVerwG, Beschl. v. 31.7.2019 - 3 B 7/18 (Bay. VGH)}

\section{Florian Wolf}

Die Entziehung der Approbation ist immer wieder Gegenstand der höchstrichterlichen Rechtsprechung, was angesichts ihrer existentiellen Bedeutung auch nicht verwundert.

Immer wieder tauchen dabei dieselben Probleme auf: Die Abgrenzung der Tatbestandsmerkmale „Unzuverlässigkeit“ und „Unwürdigkeit“, die Frage der Notwendigkeit eines prognostischen Elements, der maßgebliche Zeitpunkt, zu dem die Tatbestandsmerkmale vorliegen müssen, und schließlich der Sonderfall von Verfehlungen, die nicht im Arzt-Patienten-Verhältnis oder auch nur im Verhältnis zu Kostenträgern begründet sind, sondern Straftaten außerhalb der eigentlichen Berufsausübung betreffen.

Geradezu schulmäßig definiert das BVerwG zunächst den Begriff der Unwürdigkeit und weist auf die hohen Voraussetzungen hin, die angesichts des Verhältnismäßigkeitsgrundsatzes und der Bedeutung der Approbation an eine Widerrufsentscheidung geknüpft werden müssen. Anlass für den Widerruf kann nur ein schwerwiegendes Fehlverhalten sein, das geeignet ist, das Vertrauen der Öffentlichkeit in den ärztlichen Berufsstand so nachhaltig zu erschüttern, dass bei Würdigung aller Umstände die weitere Berufsausübung als untragbar erscheint. Dies kann auch bei Straftaten der Fall sein, die nicht im Arzt-Patienten-Verhältnis angesiedelt sind oder die ein außerberufliches Fehlverhalten betreffen.

Das BVerwG fasst weiter die ständige Rechtsprechung zusammen, dass der Begriff der Unwürdigkeit im Gegensatz zur Unzuverlässigkeit gerade kein prognostisches Element enthält. Der Approbationswiderruf erfordert keine auf die Person des Betroffenen bezogene Gefahrenprognose; Wiederholungsgefahr ist nicht erforderlich.

Besonders interessant wird die Entscheidung des BVerwG, wenn man sie mit der Entscheidung des VG

Rechtsanwalt Dr. iur. Florian Wolf,

Fachanwalt für Medizinrecht,

Praxisrecht Dr. Fürstenberg \& Partner,

Römerstr. 9, 69115 Heidelberg, Deutschland
Hamburg v. 23.2.2019, Az. 17 K 4618/18, vergleicht, welches der Anfechtungsklage eines Chefarztes stattgegeben hatte, dem die Approbation wegen Abrechnungsbetrugs gegenüber der KÄV Hamburg entzogen worden war. Dem rechtskräftigen Strafbefehl hatte das - bei ermächtigten Klinikärzten geradezu klassische - Problem des Verstoßes gegen das Gebot der persönlichen Leistungserbringung zugrunde gelegen.

Unter Heranziehung derselben Grundsätze und auch unter dem Obersatz, dass der Verstoß gegen das Gebot der persönlichen Leistungserbringung und der damit einhergehende Abrechnungsbetrug den Widerruf der Approbation rechtfertigen können, kommt das VG Hamburg zum Ergebnis, dass der Widerruf der Approbation im konkreten Fall rechtswidrig sei.

Maßgeblich für das VG Hamburg war bei dieser Entscheidung, dass sich aus der Begründung der Entscheidung nicht schlüssig ergebe, dass das Fehlverhalten des Chefarztes das Vertrauen des Patienten in eine ordnungsmäße Aufgabenerfüllung beeinträchtige. Dabei war u.a. entscheidend, dass die KÄV Hamburg während der von ihr selbst initiierten strafrechtlichen Ermittlungen dennoch eine erneute Ermächtigung erteilt hatte, was von der Approbationsbehörde nicht in die Gesamtabwägung eingestellt worden war. Auch hatte die Behörde nicht zwischen einem „normalen“ Abrechnungsbetrug im Sinne einer Abrechnung nichterbrachter Leistungen und dem vorliegenden Verstoß gegen das Gebot der persönlichen Leistungserbringung differenziert.

Die Entscheidung des BVerwG ruft insbesondere die Grundsätze zum Widerruf der Approbation und den Unterschied zwischen Unwürdigkeit und Unzuverlässigkeit ins Gedächtnis. Der Vergleich mit der Entscheidung des VG Hamburg wiederum zeigt, wie wichtig die Argumentation am Einzelfall im Rahmen der Anfechtungsklage ist: Bei Zugrundelegung derselben Obersätze und unter Heranziehung derselben hohen Maßstäbe für die Widerrufsentscheidung kommen die Gerichte zu konträren Ergebnissen. Dies zeigt, wie wichtig es ist, im rechtlichen und tatsächlichen Vortrag jeden Aspekt zu beleuchten, um in der Gesamtabwägung einen Schwerpunkt zugunsten des Arztes zu setzen.

\section{Keine Befugnis nationaler Überwachungs- behörden zum Erlass von transnationalen Verbotsverfügungen}

MPG §§2, 3, 27 Abs. 1, Abs. 2, 36; AMG §69; MedizinprodukteRichtlinie Art. 8, Art. 18; GG Art. 25

1. Die Befugnis zum Einschreiten nach $\$ 27$ Abs. 2 MPG besteht auch dann, wenn der Anwendungsbereich des Medizinproduktegesetzes nach $\mathbb{2}$ Abs. 1 bis 3 und 4a MPG nicht eröffnet ist oder die Anwendung des Medizinproduktegesetzes nach $\$ 2$ Abs. 5 MPG ausgeschlossen ist.

2. $₫ 27$ Abs. 2 MPG bietet keine taugliche Rechtsgrundlage für die Untersagungsanordnung einer deutschen Behörde mit transnationaler Wirkung für den gesamten Europäischen Wirtschaftsraum. Der räumliche Geltungsbereich des $₫ 27$ MPG ist auf das Bundesgebiet beschränkt.

Nds. OVG, Urt. v. 17.12.2019 - 13 LB 135/19 (VG Osnabrück)

Eingesandt vom Veröffentlichungsverein von Mitgliedern des Nds. OVG; bearbeitet von VRiVG Andreas Fleischfresser, Vorsitzender der 7. Kammer des VG Köln, Im Paradies 22, 51789 Lindlar, Deutschland 